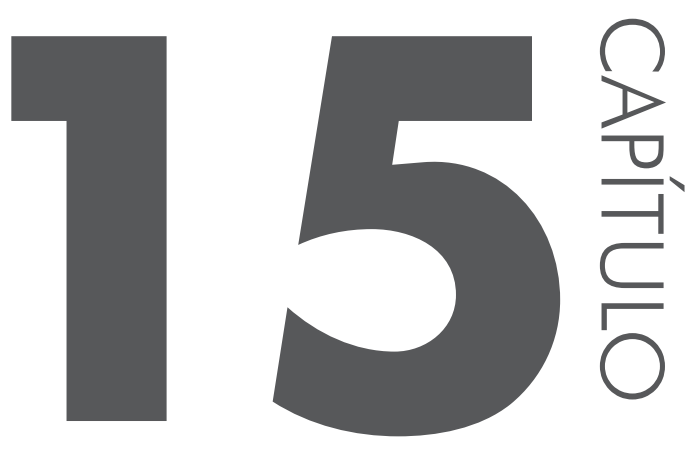

\title{
O USO DE TECNOLOGIAS DE INFORMACÃO E COMUNICAC̣Á̃O NA MEDIAC̣ÃO DA ESPECIALIZAÇÃO EM TIC APLICADAS À SEGURANÇA PÚBLICA E DIREITOS HUMANOS
}

Natana Lopes Pereira

Fernando José Spanhol Thabata Clezar de Almeida

\section{INTRODUC̣ÃO}

O desenvolvimento das tecnologias impulsionou o surgimento da cultura digital, tendo como principal característica o uso das Tecnologias da Informação e Comunicação (TIC) e propondo maior acesso ao processo de ensino aprendizagem e na construção competência virtual individual. 
Nesse contexto, destaca-se o estudo de caso realizado no curso de especialização em Tecnologias da Informação e Comunicação Aplicadas à Segurança Pública e Direitos Humanos (Pós TIC Senasp), da Universidade Federal de Santa Catarina, campus de Araranguá.

O referido curso é destinado a profissionais de segurança pública em atividade: policiais civis, policiais militares, bombeiros militares, profissionais de perícia forense, guardas municipais, policiais federais, policiais rodoviários federais, agentes penitenciários federais e estaduais, disponibilizando-se 40 vagas para os mesmos, assim como dez vagas para representantes da sociedade civil.

Pereira (2015) apud Lunardi (2013) destaca como objetivo geral do curso a capacitação de profissionais de Segurança Pública e membros da sociedade civil, na utilização de Tecnologias da Informação e Comunicação para prevenção, combate e diminuição da violência e criminalidade, bem como na defesa e na promoção dos direitos humanos no extremo sul catarinense.

Seus objetivos específicos, segundo Pereira (2015) apud Lunardi (2013), são os seguintes: capacitar 40 profissionais de Segurança Pública e dez membros da sociedade civil, e consolidar grupos interinstitucionais e de movimentos sociais com projetos de ensino, pesquisa, extensão e inovação na área de TIC aplicada à Segurança Pública e Direitos Humanos na região do extremo sul catarinense.

O curso, realizado de forma presencial, utilizou alguns sistemas computacionais disponibilizados pela Universidade Federal de Santa Catarina (UFSC) em sua execução. Visando observar a importância desses sistemas, aplicou-se um instrumento de coleta de dados, com o objetivo principal de analisar a aplicabilidade dos sistemas computacionais utilizados no decorrer do curso, além de obter uma avaliação global do curso envolvendo professores, infraestrutura, apoio extraclasse, desenvolvimento próprio, curso, biblioteca (BSARA) e restaurante universitário (RU).

Desta forma, o presente estudo objetiva apresentar, além do desempenho e aplicabilidade do curso, os sistemas e os aspectos referentes à tecnologia disponibilizados aos alunos no mesmo.

\section{FUNDAMENTAC̣ÃO}

O uso de novas TIC está cada vez mais presente no cotidiano das pessoas. A sociedade as utiliza para vários fins, como lazer, educação, trabalho, entre outros. Podem ser utilizadas por distintas faixas etárias e por diferentes classes sociais, proporcionando, segundo Andrade e Silva (2008), uma sociedade globalizada, o conhecimento de diversas culturas, a melhoria dos meios de comunicação e aumento da rapidez.

Para Silva et al. (2011), a tecnologia está tão presente em nosso cotidiano que não notamos mais que são artificiais. $\mathrm{O}$ avanço tecnológico garantiu novas 
maneiras de usar as TIC para a produção e propagação de informações, a interação e a comunicação em tempo real, contribuindo, assim, para o surgimento das Tecnologias de Informação e Comunicação.

A cada instante surge um novo recurso tecnológico, para um ou diversos fins, visando facilitar ou auxiliar em diversas ou determinada atividade.

Nesse contexto, Rosseti e Morales (2007) destacam que essas novas informações, comunicação e tecnologias computacionais estão mudando a organização e o conteúdo de trabalho; enfatizando, em certa escala, que carreiras de trabalho de longa vida estão se transformando em um mosaico, no qual o trabalho produtivo, o aprendizado e o desenvolvimento de competência são inseparáveis. Os autores consideram, ainda, que elas favorecem a renovação em redes informais de aquisição e de troca de conhecimento, além de facilitar o compartilhamento de problemas, perspectivas, ideias e soluções em seu dia a dia profissional.

Este avanço tecnológico molda a sociedade, em constante busca por informação e conhecimento. Desta forma, é de extrema importância o investimento das organizações em capacitações nas mais diversas áreas. Nesse contexto, visando principalmente qualificar profissionais de segurança pública, instituiu-se a Rede Nacional de Estudos em Segurança Pública, a ser implementada pela Secretaria Nacional de Segurança Pública (Senasp), por meio do Departamento de Pesquisa, Análise de Informação e Desenvolvimento de Pessoal em Segurança Pública (Depaid).

A Renaesp é um projeto de educação permanente, voltado aos profissionais de segurança pública, e também aos interessados e atuantes nesta área. A rede constitui-se a partir da parceria com Instituições de Ensino Superior (IES), que promovem cursos de pós-graduação lato sensu, na modalidade presencial, sobre diferentes temas relacionados à Segurança Pública (BRASIL, 2015 apud MIKI, 2015)

A proposta do curso de especialização em Tecnologias da Informação e Comunicação Aplicadas à Segurança Pública e Direitos Humanos foi aprovada pelo Ministério da Justiça, por meio do Edital de Chamada Pública $n^{\circ}$ 6, de 21 de junho de 2012. Este edital selecionou propostas referentes a projetos de curso de pós-graduação lato sensu, especialização em segurança pública, com o escopo de estabelecer parceria que se efetivasse por meio de Termo de Cooperação. O objetivo de edital proposto foi a oferta por instituições federais de ensino superior de cursos de pós-graduação lato sensu (especialização), na modalidade presencial, destinados a profissionais da segurança pública da ativa, tendo por referência a Matriz Curricular Nacional nas áreas temáticas informadas no edital. A proposta conteve-se nas temáticas de gestão de conhecimento, informações sobre violência e criminalidade, e Segurança Pública e Direitos Humanos. 


\section{PROCEDIMENTOS METODOLÓGICOS}

Com o objetivo de analisar a importância dos sistemas computacionais utilizados no curso, o procedimento metodológico adotado nesta pesquisa caracteriza-se como descritivo, predominantemente qualitativo, documental e bibliográfico.

A pesquisa bibliográfica realizada por meio de leitura e análise de periódicos e livros, segundo Gil (2010), é desenvolvida mediante material já elaborado, principalmente livros, artigos científicos revistas, teses, entre outros. Permite ao investigador a cobertura de uma gama de fenômenos muito mais ampla do que poderia pesquisar diretamente.

Para poder identificar os benefícios e as dificuldades encontradas na execução do projeto foi adotada a pesquisa descritiva que, de acordo com Lakatos e Marconi (2010, p.170) “apresenta como principal finalidade o delineamento ou análise das características de fatos ou fenômenos, a avaliação de programas, ou o isolamento de variáveis principais ou chave".

Como a pesquisa abrangia dados de difícil mensuração, utilizou-se da pesquisa quantitativa, que, segundo Dalvofo, Lana e Silveira (2008) apud Richardson (1989), é uma forma adequada para entender e analisar dados de difícil mensuração como motivações e dificuldades pessoais.

Em relação aos meios, caracteriza-se, segundo Lakatos e Marconi (2010, p.157), como pesquisa documental pela fonte de coleta de dados constituída de fontes primárias como documentos internos, arquivos virtuais e físicos com dados sobre o curso, website institucional, relatórios e outros documentos.

\subsection{Coleta de Dados}

Quanto à coleta de dados, realizou-se pesquisa aplicada aos alunos do curso de especialização em Tecnologias da Informação e Comunicação Aplicadas à Segurança Pública e Direitos Humanos.

A pesquisa, composta por 61 questões, abrangia avaliação sobre: docentes, infraestrutura, apoio extraclasse, desenvolvimento próprio, o curso, Moodle, CAPG, ID UFSC-SETIC, biblioteca e restaurante universitário.

$\mathrm{O}$ instrumento para coleta de dados utilizado foi o Formulário Google Drive. Ele organiza, de forma automática em Planilhas Google as informações dos dados coletados, possibilitando a visualização dos dados por meio de gráficos.

Segundo Pereira (2015, p. 35), o "software utilizado para elaboração e aplicação da pesquisa possibilita o armazenamento de arquivos na nuvem, podendo estes ser compartilhados com quem o usuário disponibilizar o acesso". 
Para a análise dos dados, utilizou-se itens de múltipla escolha, sendo disponibilizada a seguinte escala de satisfação: Ótimo, Bom, Regular, Ruim ou Péssimo, correspondendo aos valores $5,4,3,2,1$, respectivamente.

Além dos temas de múltipla escolha, foi aplicada uma questão descritiva, com predominância da análise qualitativa.

\section{AMBIENTES COMPUTACIONAIS DA UFSC}

A Universidade Federal de Santa Catarina utiliza diversos ambientes computacionais, visando à melhoria na gestão administrativa e acadêmica, refletindo em um sistema organizado, eficiente e consistente.

Nesse sentido, Pereira (2015) lista como sistemas de gestão acadêmica:

Quadro 15.1 Sistemas de gestão acadêmica

\begin{tabular}{c|c} 
CAPG - Controle Acadêmico de Pós-Graduação \\
CAGR - Controle Acadêmico de Graduação \\
Gestão Acadêmica \\
$\begin{array}{c}\text { MOODLE - Modular Object Oriented Distance Learning } \\
\text { BSARA - Biblioteca Universitária da Universidade Federal de } \\
\text { Santa Catarina }\end{array}$ \\
IdUFSC - Sistema de Gestão de Identidade da UFSC \\
\hline
\end{tabular}

Os sistemas de gestão administrativa, criados para servir de suporte técnico ao propósito de reduzir o uso de papel nas rotinas administrativas da instituição, são os seguintes (PEREIRA, 2015):

Quadro 15.2 Sistemas de gestão administrativa

\begin{tabular}{l|c}
\hline ALX - Sistema de Gestão de Materiais \\
Gestão Administrativa & SCL - Sistema de Gestão de Compras e Licitações \\
SIP - Sistema de Gestão Patrimonial \\
SPA - Sistema de Processos Administrativos \\
SCL - Sistema de Gestão de Compras e Licitações \\
\hline
\end{tabular}

\section{AMBIENTES UTILIZADOS}

Além da avaliação sobre docentes, infraestrutura, apoio extraclasse, desenvolvimento próprio e avaliação do curso, a pesquisa também analisou os seguin- 
tes sistemas computacionais da área de gestão acadêmica utilizados no decorrer do curso:

a) CAPG (Controle Acadêmico de Pós-Graduação).

b) MOODLE (Modular Object Oriented Distance Learning).

c) IdUFSC (Sistema de Gestão de Identidade da UFSC) e serviços SETIC.

d) BSARA (Biblioteca Universitária da Universidade Federal de Santa Catarina - Campus Araranguá).

\section{RESULTADO DA PESQUISA APLICADA}

Visando à análise sobre os elementos que regem o curso, em especial sobre os sistemas computacionais utilizados, aplicou-se instrumento de coleta de dados aos alunos do curso de especialização em Tecnologias da Informação e Comunicação Aplicadas à Segurança Pública e Direitos Humanos para avaliar o índice de satisfação sobre os itens analisados.

A pesquisa foi enviada aos 33 alunos do curso, obtendo retorno de 20 acadêmicos. Constatou-se que todos os itens pesquisados obtiveram maior porcentagem nos índices: Ótimo e Bom, sendo interpretado como um indicativo da positiva execução do curso.

A partir da análise dos dados coletados, referente a professores, infraestrutura, apoio extraclasse, desenvolvimento próprio e curso, pode-se constatar que:

a) aulas planejadas de forma clara e objetiva, com dinâmicas predominantemente expositivas, sendo estas realizadas por professores qualificados, com alto nível de conhecimento;

b) o incentivo pelos docentes aos discentes na publicação de artigos;

c) infraestrutura com espaço, iluminação e equipamentos adequados;

d) avaliação positiva ao desempenho da coordenação do curso;

e) predominância da escala de satisfação Ótima referente à avaliação do curso, sendo de extrema importância ao desempenho profissional dos alunos;

f) ótimo desempenho dos professores, apresentando proposta curricular equilibrada;

g) material didático mais utilizado: livros, artigos e periódicos;

h) dificuldades no curso em relação ao conteúdo, mas não em relação à condução do mesmo pelo docente.

Em relação aos sistemas acadêmicos utilizados pelos alunos (MOODLE e CAPG), a pesquisa apontou os seguintes índices:

a) utilização do ambiente virtual de aprendizagem Moodle, sendo constatado baixo grau de dificuldade em utilizar os recursos disponibilizados; 
b) frequência de acesso ao Moodle, pelos acadêmicos, duas a três vezes por semana;

c) prática frequente de exercícios, por meio de recursos disponibilizados do Moodle;

d) dificuldade de acesso ao CAPG relacionado à falta de informação sobre sua funcionalidade;

e) frequência de acesso ao CAPG com predominância mensal e quinzenalmente.

O instrumento de avaliação aplicado apresentou um positivo índice de aprovação ao curso e todos seus fatores relacionados. Destaca-se, entre vários itens, a avaliação dos acadêmicos em relação ao nível de conhecimento e qualificação didática do corpo docente.

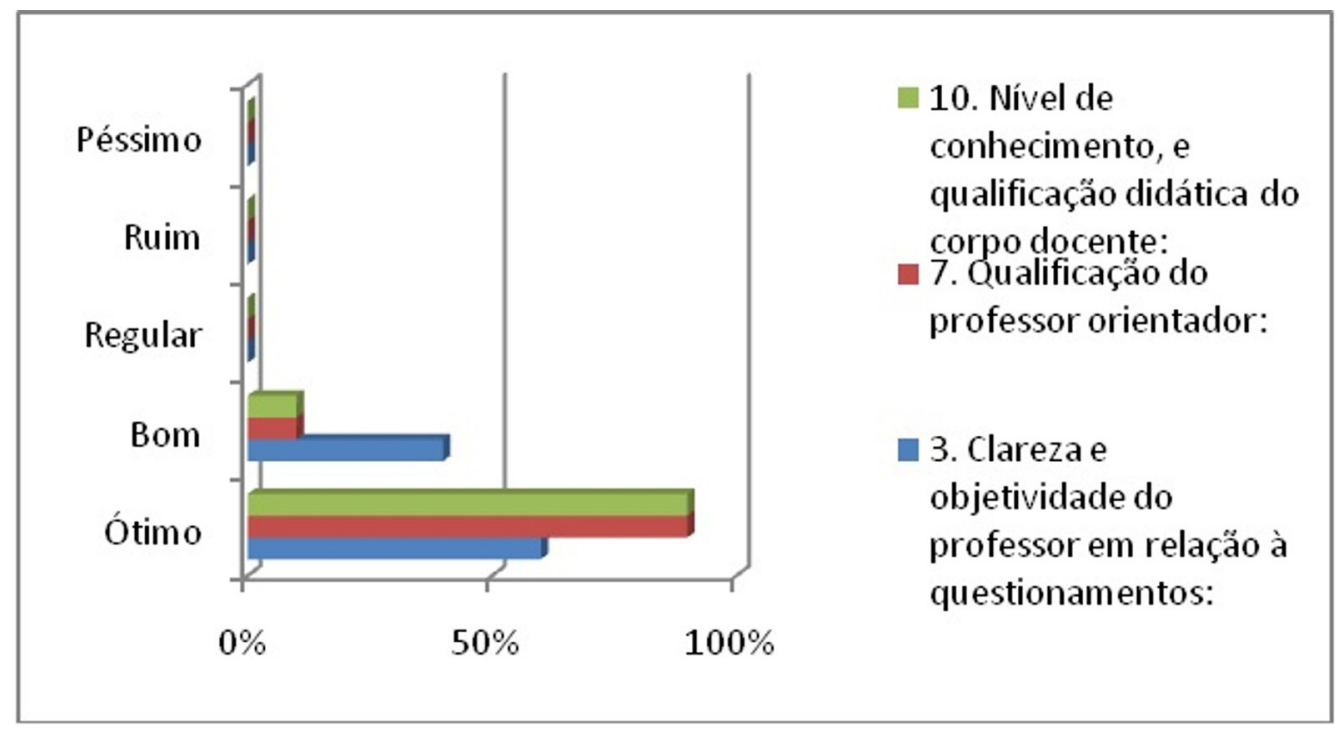

Figura 15.1 Resultado da pesquisa - professores.

\section{CONCLUSÃO}

O curso de especialização em Tecnologias da Informação e Comunicação Aplicadas à Segurança Pública e Direitos Humanos proporcionou aos alunos, por meio de formação acadêmica, o aprimoramento do conhecimento sobre TIC.

As temáticas abrangidas pela proposta do curso são de extrema importância, refletindo maior eficiência e eficácia, maior interatividade e o compartilhamento de informações nas atividades profissionais desenvolvidas pelos alunos. 
Com esta pesquisa, pode-se perceber a importância dos sistemas computacionais utilizados na execução do curso, tanto na área de gestão acadêmica, quanto na área de gestão administrativa, que proporcionaram mais qualidade ao ensino dos alunos, destacando-se principalmente o uso do Ambiente Virtual de Ensino e Aprendizagem (AVEA) MOODLE customizado para o curso.

De acordo com os dados coletados, o acesso frequente ao AVEA e a prática constante na utilização de seus recursos interferiram de forma positiva no processo de ensino aprendizagem. Esse ambiente virtual permitiu a integração de distintas mídias, recursos, além das informações apresentadas de maneira organizada, com o intuito de facilitar a interação entre o aluno e o conhecimento proposto pelo curso.

Por fim, evidencia-se, além da importância da proposta do curso de especialização em Tecnologias da Informação e Comunicação Aplicadas à Segurança Pública e Direitos Humanos, a relevância de cada item analisado e suas atribuições para resultados satisfatórios do mesmo possam ser repetidos para registro de futuras pesquisas.

\section{REFERÊNCIAS}

ALVES, R. M. Gestão educacional e novas tecnologias da informação comunicação: atualizações necessárias disponíveis para a cultura educacional. Revista PUC-SP, São Paulo, v. 12, n. 2, 2014.

ANDRADE, L. M.; SILVA, F. C. Tecnologias de Informação e Comunicação: as influências das novas tecnologias perante a sociedade. Associação de Leitura do Brasil, São Paulo, 2008. Disponível em: <http://alb.com.br/arquivo-morto/anais-jornal/jornal4/ comunicacoesPDF/62_tecnologiasFABIANO.pdf>. Acesso em: 15 ago. 2015.

BRASIL. Ministério da Justiça. Secretaria Nacional de Segurança Pública. MJ oferece vagas para pós-graduações em segurança pública. Brasília, DF, 2015. Disponível em:<http://www.justica.gov.br/noticias/mj-oferece-vagas-para-pos-graduacoes-emseguranca-publica-oferecem-250-vagas-em-cinco-estados/>. Acesso em: 9 ago. 2015. CORREIA, R. L.; SANTOS, J. G. A importância da Tecnologia da Informação e Comunicação (TIC) na Educação a Distância (EAD) do Ensino Superior (IES). Revista Aprendizagem em EAD, Distrito Federal, v. 2, n. 1, 2013. Disponível em: <http:// portalrevistas.ucb.br/index.php/raead/article/viewFile/4399/2899>. Acesso em: 30 jun. 2015. DALFOVO, M. S.; LANA, R. A.; SILVEIRA, A. Métodos quantitativos e qualitativos: um resgate teórico. Revista Interdisciplinar Científica Aplicada. Blumenau, v. 2, n. 4, p. 1-13, 2008. Disponível em: <http://www.unisc.br/portal/upload/com_arquivo/metodos_ quantitativos_e_qualitativos_um_resgate_teorico.pdf >. Acesso em: 02 jul. 2015.

GIL, A.C. Como elaborar projetos de pesquisa. 5. ed. São Paulo: Atlas, 2010.

LAKATOS, E. M.; MARCONI, M. A. Fundamentos da metodologia científica. 7. ed. São Paulo: Atlas, 2010. 
LUNARDI, G. M. Processo 23080.003511/2013-69. Araranguá: Universidade Federal de Santa Catarina, 2013.

NAKAYAMA, M. K. et al. A atuação da Universidade Federal de Santa Catarina no Programa Aluno Integrado: um projeto de extensão. Florianópolis: UFSC, 2010. Disponível em: <https://repositorio.ufsc.br/bitstream/handle/123456789/96862/A\%20 ATUA $\%$ C3\% $\% 7 \%$ C3\% 83O\%20DA \%20UNIVERSIDADE \%20FEDERAL \%20DE \% 20 SANTA \%20CATARINA\%20NO\%20PROGR.pdf?sequence=1>. Acesso em: 28 jun. 2015.

PACHECO, A. S. V. et al. Ambientes computacionais e Tecnologias da Informação para a gestão do conhecimento na Universidade Federal de Santa Catarina. Florianópolis, 2009. PEREIRA, D. M.; SILVA, G. S. As Tecnologias de Informação e Comunicação (TICs) como aliadas para o desenvolvimento. Caderno de Ciências Sociais Aplicadas. Bahia, n. 10, 2010. Disponível em: <http://periodicos.uesb.br/index.php/cadernosdeciencias/ article/viewFile/884/891>. Acesso em: 03 jul. 2015.

PEREIRA, N. L. Avaliação dos sistemas computacionais utilizados no curso Pós TIC SENASP- UFSC. Araranguá. 2015. 55 p. (Relatório de Estágio II - UFSC)

ROSSETI, A.; MORALES, A. B. O papel da tecnologia da informação e na gestão do conhecimento. Revista Ciência da Informação, Brasília, DF, v. 36, n. 1, p. 124-135, jan.abr., 2007. Disponível em: <http://revista.ibict.br/ciinf/article/view/1191/1363>. Acesso em: 18 jun. 2015.

SILVA, E. R. G.; OLIVEIRA, T. P. S.; ARAÚJO, T. S.; ROVER, A. J. Sistema integrado de gestão da informação para segurança pública. $3^{\text {a }}$ Conferência Ibérica de Sistemas e Tecnologias de Informação. Vigo, Espanha, 2008. Disponível em: <http://revistapos. cruzeirodosul.edu.br/index.php/epd/article/viewFile/511/436>. Acesso em: 20 jun. 2015. SILVA, J. F.; SCHIMIGUEL, J. O uso das TICs no ensino superior: a integração de diferentes tecnologias à educação estatística. Encontro de Produção Discente PUCSP/Cruzeiro do Sul, São Paulo, v.1, n.1, 2012. Disponível em <http://revistapos. cruzeirodosul.edu.br/index.php/epd/article/viewFile/511/436>. Acesso em: 20 abr. 2015. SILVA, R. P. Educação e segurança pública: uma perspectiva de cidadania e Direitos Humanos. Revista Espaço Acadêmico. v.11, n.125, 2011. Disponível em: <http://www. periodicos.uem.br/>. Acesso em: 20 abr. 2015.

UNIVERSIDADE FEDERAL DE SANTA CATARINA. Sistemas de Gestão Adimistrativa. Florianópolis, 2015. Disponível em: <http://egestao.ufsc.br/>. Acesso em: 16 maio. 2016. 
